\title{
¿QUE SIGNIFICADO LE ASIGNAN UN GRUPO DE MUJERES A LAS \\ ACTIVIDADES PARA Sí MISMAS?
}

\section{¿HOW MEANINGFUL IS FOR THE WOMEN THEMSELVES THE ACTIVITIES THEY CARRY OUT?}

\author{
Paula Torrico Mayol, Daniela Urrutia García ${ }^{\mathrm{i}}$, Marjorie Schliebener Tobar ${ }^{\mathrm{iii}}$
}

\section{Resumen}

Cambios notorios en el estilo de vida de las mujeres de Santiago de Chile, es de lo que da cuenta una encuesta realizada, en julio de 2007 a 450 mujeres que trabajan fuera del hogar y además cumplen con las tareas domesticas y cuidado de la familia. En este estudio, la mayoría de las mujeres capitalinas se perciben estresadas $(60 \%)$ por estas responsabilidades y por el escaso tiempo dedicado a si mismas.

En el marco de la ciencia de la ocupación, basada en utilizar la actividad con propósito como medio hacia la comprensión del sentido de su vida; es que por medio de un investigación cualitativa, con una entrevistas semi estructurada, de 7 preguntas abiertas en relación a las actividades realizadas para si mismas. Con las preguntas se explora entorno a la forma, función y significado de las actividades.

Los resultados indican que las entrevistadas consideran importante realizar actividades para si mismas, pero en la practica no lo hacen, (75\%). Reconocen haber las tenido (100\%), pero haberlas abandonado debido al trabajo aumento de la responsabilidades familiares o por percibirlas finalmente como una obligación mas (75\%). Sus familias no se opone a que las realicen pero tampoco facilitan su ejecución.

Conclusión: Se pudo constatar un fuerte desequilibrio en el patrón ocupacional de nuestras entrevistadas, lo cual convierte esta situación en un factor de riesgo y alienación ocupacional.

Palabras claves: ocupación, significado, Actividades para si mismas, calidad de vida.

\footnotetext{
${ }^{\text {i }}$ Terapeuta Ocupacional, Clínica UC San Carlos de Apoquindo. Teléfono:7548874, Camino El Alba 12351, Las Condes, E-mail: ptorrico@med.puc.cl

ii Terapeuta Ocupacional, docente escuela de Terapia Ocupacional de la Universidad Austral de Chile. Teléfono:63-2935668, Campus Isla Teja s/n casa 2 tipo A, e-mail diurutia@uach.cl

iii Licenciada en Ciencia de la Ocupación, estudiante Terapia Ocupacional. marjarate@gmail.com
} 


\begin{abstract}
A study done in July 2007 among 450 women in Santiago, Chile showed there has been enormous life style changes for women who work outside the home as well as take charge of domestic chores including caring for their families. The study showed that the majority (60\%) of the women interviewed were suffering from stress as a result of these responsibilities and the lack of time they had to dedicate to their own needs.
\end{abstract}

Within the context of occupational science and based on using this activity as way of better understanding their lives; done as a qualitative research, with loosely-structured interviews consisting of 7 open questions relating to the work carried out by themselves. The questions were an exploration of their family life, their role in it and the significance of their activities.

Results show that the interviewees consider that it is important to carry out activities for their own benefit, but, at the end of the day, they don't (75\%).They do admit to having had them in the past $(100 \%)$, but they abandoned them due to a job, more family responsibilities o for seeing it as an additional obligation (75\%). Their families don't object to them doing those duties but they don't contribute to making them possible either.

Conclusion: We could confirm a strong imbalance in the occupational pattern of the interviewees, which turns this into a risk situation and occupational alienation.

Key Words: Occupation, Meaning, Activity to herself, Quality of Life. 


\section{INTRODUCCION}

Actualmente en nuestro país existe un gran número de mujeres que desempeñan paralelamente actividades laborales con actividades del cuidado del hogar y la familia.

Estudios recientes sobre la distribución de roles y estrés(1) ponen de manifiesto que la fuerte carga de responsabilidades y la superposición de ocupaciones que tienen las mujeres chilenas influyen en su percepción de estrés (de las mujeres encuestadas en este estudio un 30,4\% percibe niveles medio de estrés y $29,7 \%$ percibe altos niveles de estrés)

Las mujeres chilenas se reconocen cada vez mas estresadas y refieren que dedican un tiempo excesivo al trabajo, los hijos y la administración del hogar en desmedro del tiempo para realizar actividades para si mismas.

Es en este punto, como terapeutas ocupacionales, que consideramos importante para nuestra disciplina conocer la percepción que tienen las mujeres chilenas acerca de las actividades para si mismas, ya que estamos convencidas que un desequilibrio en las diferentes ocupaciones influyen no solo en bienestar físico y mental sino en la calidad de vida de las mujeres - desde esta mirada es necesario conocer no solo lo que esta pasando con las mujeres chilenas, sino mas bien, lo que ocurre con la mujer dentro del contexto actual latinoamericano, e investigar desde la ciencia de la ocupación, ya que como hemos observado los ritmos de vida, la presión del tiempo, y las distintas ocupaciones que desempeñamos apuntan a aumentar la productividad en los distintos contextos, y sobre todo a cumplir, no solo en el trabajo sino en la familia, lo cual provoca impactos en los niveles de satisfacción personal.

Es importante referirnos a un estudio realizado, dirigido por Carlos Catalán(2) "El uso del tiempo y el consumo de medios", el cual hace referencia a que en Chile una persona dispone de un promedio de 5 horas y 6 minutos de tiempo libre diario. Esta es una situación distinta a la de Italia o Australia, por ejemplo, donde cada trabajador dedica como promedio 6 horas diarias al ocio; Esta diferencia, que a primera vista podría parecer poco significativa, cobra su verdadero peso cuando se consideran las horas acumuladas en el tiempo, en una semana, aproximadamente 6 horas, lo que constituye casi una jornada de trabajo, esto se ve reflejado en las diferentes tendencias que tienen las jornadas laborales a nivel mundial, en un estudio realizado en 240 países (ILO 1999) señala que en Europa la tendencia apunta a disminuir la jornada laboral en privilegio de aumentar las horas de tiempo libre y destinado a la familia, ejemplo de esto se puede observar en Francia o Alemania donde las jornadas se han limitado por ley a 35 horas semanales, lo que en 1997 se materializaba en 1.656 horas anuales de trabajo. En contraste con Latinoamérica y el Caribe donde se trabaja entre 1.800 y 2.000 horas al año. En Chile, lugar en que se centra esta reflexión, la encuesta laboral de la Dirección del 
Trabajo, realizada en 1999, indica que las horas anuales de trabajadores dependientes es de 2.517,84, mientras la encuesta de INE, del último trimestre de 1999, da cuenta de 2.260,44 horas anuales (ENCLA99).

En relación a los datos anteriores es importante considerar que la mujer Chilena se ha introducido en estas jornadas laborales extensas, y por consiguiente el promedio de 5 horas que tienen las mujeres para destinarlas al uso del tiempo libre, es utilizado en una gran cantidad de actividades ya comprometidas, principalmente actividades domésticas y familiares que inundan este tiempo libre.

Por lo cual es importante para nosotros conocer la importancia le asignan las mujeres chilenas a este tipo de actividades y como estas pasan a formar parte de la orquestación de sus actividades.

El presente trabajo pretende buscar respuestas a muchas interrogantes y ser un aporte no solo a la vida de las mujeres consultadas, sino un aporte desde la ocupación a la salud y calidad de vida de muchas mujeres de Chile y por qué no de Latinoamérica.

\section{MARCO CONCEPTUAL}

La ciencia de la ocupación y su practica parten de la premisa que el ser humano es un ser ocupacional, que tiene la capacidad de utilizar la actividad con propósito como medio hacia la comprensión del sentido de su vida(3). Wilcock refiere que el ser humano posee una necesidad de ocuparse, hacia la satisfacción de tres funciones principales que ésta le brindaría: "1) proveer necesidades corporales inmediatas de sustento, autocuidado, refugio, y seguridad; 2) desarrollo de estructuras sociales y técnicas, y tecnología apuntada a la superioridad sobre predadores y el medioambiente; 3) ejercicio y desarrollo de las capacidades personales permitiendo al organismo ser sostenido y crecer(4) Estos aspectos resultan coherentes con el objetivo de este trabajo ya que la mujer Chilena y Latinoamericana han ido evolucionando en distintas ocupaciones, pero estas ocupaciones, ¿nacen de satisfacer sus propias necesidades o están determinadas por el contexto histórico y cultural en el cual están inmersas? Por esto es necesario realizar una revisión de cómo la mujer en el ámbito latinoamericano ha ido determinando sus ocupaciones, como el género, la edad, incluso la raza nos ha determinado para cumplir ocupaciones que están casi siempre en relación a los otros.

Desde la época de la colonia las mujeres blancas realizaban funciones diferentes en contraste con las indígenas, negras, y mulatas.(5) 
Las primeras se quedaron el hogar con el objetivo de criar a los hijos, reproduciendo de esta manera el sistema colonial.

Los matrimonios eran un rito sin amor ni consenso. Solo garantizaba la descendencia y la herencia a los hijos legítimos, continuando con el sistema patriarcal.

Las separaciones eran infrecuentes en las familias españolas y criollas. Había que mantener una imagen externa de matrimonio feliz y permanente.

La mujer soltera que pasaba los 25 años, era relegada a la casa paterna o a la de una hermana para cuidar sobrinos o ancianos.

La sexualidad de la mujer fue construida por la Iglesia Católica, solo dentro del matrimonio era permitido y siempre con el fin de la procreación.

Los valores de la mujer blanca, desde la óptica masculina, estaban puestos en su belleza, su condición de madre y fiel esposa.

La mujer negra impuso otras características, dio la fuerza del trabajo; estaba destinada a la minería y plantación: Recién a fines de la Colonia se ocupo esencialmente de las tareas domesticas en las casas señoriales.

Para comprender la historia de la mujer en Latinoamérica, tenemos que analizar las características de la mujer indígena, las costumbres, la moral, la manera de subordinarse al hombre y la participación en el trabajo comunitario.

- Descendencia matrilineal, oponiéndose al tipo de familia patriarcal impuesto por la colonia.

- La mujer aborigen producían lo que consumían.

- Una mujer soltera podía tener uno o más hijos, sin tener mayor dificultad en encontrar un compañero, ellas podían usar su cuerpo libremente, a diferencia del concepto de virginidad europeo.

Durante la Colonia(6) se va conformando el ideal machista, acerca de cómo debía ser una mujer: delicada, femenina, necesitada de protección, madre ejemplar esposa sumisa y sobreprotectora de los ancianos. Con esta concepción aparece una subcultura femenina de adaptación y dependencia, fortaleciendo el sistema patriarcal.

La mujer en el Chile de hoy según Sonia Montecinos, antropóloga, en entrevista al Diario el Mercurio(7) señala. "Para las mujeres la identidad no es un problema, entendiendo que las mujeres veían la identidad materna como algo completamente asumido: su destino era ser madre. Pero en el momento en que la mujer chilena se incorpora al trabajo, se amplían las responsabilidades de identidades. Las mujeres ingresan al espacio de lo publico pero no hay una transformación social de esos espacios”. Esto coincide con lo investigado en el sentido 
que las mujeres que trabajan fuera de su casa, reconocen que no reciben mas ayuda o no comparten la responsabilidad del hogar los otros integrantes de la familia, una de nuestras entrevistadas dice: "si yo le pido a mi mama que se quede con mis niños porque tengo que ir al doctor, me ayuda, si le pido lo mismo porque tengo una fiesta me pon mala cara”.

Mas adelante dice: "El mundo de lo domestico está desvalorizado y lo que se valora es el mundo de lo publico, entonces la identidad se construye en el mundo de lo publico."Una de nuestras entrevistadas nos comenta: "si mis jefes me pide que llegue mas temprano o que me quede hasta mas tarde, yo no tengo ningún problema. Mi hijo me reclama, ... pero soy trabajolica."

Las mujeres justifican la permanencia en el ámbito de lo domestico. Con la idea de dar afecto, de que el trabajo en casa es una manera de expresar afecto. Cocinar, planchar, lavar, tiene una dimensión afectiva para la mujer." De esta perspectiva podemos decir que la mujer en la medida que se va incluyendo en actividades laborales siente que deja de aportar en al ámbito familiar, la mujer se ve inmersa en dos mundos, y es en estos dos mundos donde tiene que responder, cumplir y mediar, en desmedro del tiempo disponible para buscar las formas más apropiadas de satisfacer sus propias necesidades.

El prototipo de mujer latinoamericana se fue conformando desde hace quinientos años hasta la actualidad. Dentro este prototipo de mujer, las ocupaciones que se han ido adquiriendo están compuestas de forma, función y significado las cuales se encuentran dentro de un contexto temporal y socio cultural que las determina.

Desde sus estudios e investigaciones, Larson, Wood y F. Clark(8), concluyen que el concepto de ocupación es el resultado de la interacción de tres elementos que la componen: entendiendo como forma a un sistema objetivo de circunstancias, independiente y externo a una persona. "La realidad sociocultural de una forma ocupacional depende del consenso del grupo, pero este consenso es rara vez unánime, mas aun, el individuo que se enfrenta a una forma ocupacional puede o no estar familiarizado con sus normas y procesos socioculturalmente definidos"(9)

Se entiende la función; como el hacer, la acción, el comportamiento activo o las respuestas activas exhibidas en el contexto de una forma ocupacional.

Y el significado, concepto relevante para nuestro estudio, nace desde cómo se realiza la actividad hasta cual es la importancia que la persona le asigna a la actividad según la vivencia de ésta, el termino significado se utiliza para etiquetar la interpretación individual de una forma ocupacional, se refiere al sentido perceptivo como también a las asociaciones cognoscitivas propias de cada individuo. El significado es uno de los elementos que más 
caracteriza el concepto de ocupación y se relaciona con la potencialidad saludable de la realización. Dentro de este aspecto el significado, de la ocupación se define como "la experiencia subjetiva de la participación de ocupaciones"(10) donde se involucran diversos factores que caracterizan este concepto y que han sido objetos de estudio en diversas investigaciones, factores tales como: relación de significado e identidad, la influencia del contexto social y cultural en el significado, la capacidad de elección de la actividad, entre otras.

La posibilidad de desarrollar actividades con significado se relaciona con un aumento del bienestar y de la calidad de vida, estos quehaceres deberán dirigirse a la satisfacción de necesidades tanto básicas, sociales y también personales. Pero ¿qué sucede cuando las ocupaciones que se realizan están dirigidas, la mayor parte del tiempo, hacia "otros" y no se realizan actividades orientadas hacia sí mismo? ¿Cuales son las consecuencias de una ausencia de quehaceres que se encuentran fuera del tiempo de las actividades "obligatorias", tanto como el trabajo o la administración del hogar?

Esta es una situación que se encuentra muy presente en la mayoría de las mujeres de Santiago que trabajan, realizan los quehaceres del hogar y además cumplen con su función de madre. En un reciente estudio, realizado por Adimark, la Clínica Las Condes y Comunidad Mujer(11) llamado "Distribución de Roles y Stress" arrojó que el 60\% de las mujeres santiaguinas se sienten estresadas por su carga de responsabilidades, demostrando que solo el $47,6 \%$ de ellas percibe que le dedica menos tiempo de lo que quisieran para realizar actividades para sí mismas, ya que sus prioridades en cuanto a los quehaceres se centran principalmente en el trabajo, en el cuidado de los hijos, en los quehaceres del hogar y en el cuidado de otros, tales como madre o padre, dejando en ultimo lugar un espacio para el beneficio y crecimiento personal. Otra conclusión que también afirma esta realidad es que una de las actividades que las mujeres encuestadas refirieron que les proporcionaba satisfacción era "estar solas", es decir, tener algún tiempo sin tener que ser responsable de otros. El 67,3\% refirió sentirse responsable de "todo" y el 60\% sentirse estresadas por esta situación. Como podemos ver nos encontramos frente a una realidad potencialmente y quizás en la mayoría realmente, perjudicial para la salud, bienestar y calidad de vida de estas mujeres. 
Frente a esta situación buscamos conocer:

- Cual será la percepción de estas mujeres de las actividades para sí mismas, queremos saber si ellas pueden elegir el realizarlas o no, y que factores influyen o determinan esta situación. Y principalmente si esto se ve reflejado en la calidad de vida de estas mujeres y sus familias.

Si bien, en el ámbito individual aumentan la cantidad de factores que influyen en esta situación, también existen factores generales que se expresan a nivel personal en la vida de cada una de estas mujeres y que desencadenan esta suerte de privación de actividades para el beneficio y crecimiento personal. Estos factores generales pueden ser tanto económicos, sociopolíticos y/o culturales y podrían afectar en la elección de las ocupaciones así como también las máximas satisfacciones de la vida, tales como el desarrollo personal, el uso de las propias potencialidades y la auto-realización (necesidades de auto-realización según Maslow.) En este caso podemos ver, que se expresa fuertemente una influencia cultural sobre las funciones divididas en género, donde el hombre es responsable principalmente del trabajo y sustento económico, y la mujer del cuidado del hogar y los hijos. Frente a esta situación la mujer ha buscado una igualdad de derechos y oportunidades, esto se ve expresado en que cada año aumenta el porcentaje de mujeres que se insertan activamente al mundo laboral, pero sin dejar de lado su "otras responsabilidades culturales", hecho que se manifiesta que en un $60 \%$ de las mujeres que se encuentran dentro de esta realidad se perciban estresadas, afectando negativamente su salud y calidad de vida. Como podemos darnos cuenta, este impacto negativo de la salud y el bienestar no se relaciona directamente con alguna patología orgánica, sino más bien con factores de riesgo ocupacional, tales como la privación, la marginalización o alienación ocupacional, construyendo así un desbalance o desequilibrio ocupacional que disminuiría la sensación de salud y bienestar de estas mujeres.

\section{METODOLOGIA}

\subsection{Tipo de estudio}

El estudio según el nivel de medición y análisis de la información es de tipo cualitativo. La investigación cualitativa es aquella donde se estudia la experiencia que la persona deriva en este caso de sus actividades. La misma procura analizar exhaustivamente, con sumo detalle, la 
experiencia personal de un grupo de mujeres chilenas en relación a un asunto o actividad en particular.

A diferencia de los estudios descriptivos, correlaciónales o experimentales, más que determinar la relación de causa y efectos entre dos o más variables, la investigación cualitativa se interesa más en saber cómo se da la dinámica o cómo ocurre el proceso de en que se da el asunto o problema.

\subsection{Población de estudio}

4 mujeres de Santiago, entre 30 y 45 años, que comparten labores de dueñas de casa y a demás trabajan fuera del hogar y refieren percibirse como estresadas. Descripción de mujeres C.H, 38 años, Consejera de belleza, casada, 2 hijas (9 años y 18 años.)

A.M, 31 años, Podóloga, casada, 3 hijos. (12 años, 7 años y 9 meses.)

Z.O, 38 años, Kinesióloga, convive, 1 hijo. (14 años.)

X.M, 42 años, asistente de programación, separada hace 4 años, 2 hijos (15 y 10 años.)

\subsection{Objetivo general del estudio.}

Conocer el significado que le asignan un grupo de mujeres entre 30 y 49 años que son dueñas de casa y trabajan a las actividades para si mismas.

Para este estudio las actividades para si mismas son descritas como:

-Realizadas en tiempo no obligatorio.

-Que tenga significado para ella.

-Puede ser realizada en forma individual o grupal.

-Realizadas dentro o fuera de la casa.

-Actividades practicadas con frecuencia diaria o semanal.

Y el significado es descrito como:

-Elegido libremente

-Reporta satisfacción, es gratificante.

-Con propósito.

1.4 Objetivos específicos del estudio.

Identificar que actividades realiza para si mismas. 
Identificar con que frecuencia realiza actividades para su satisfacción personal.

Identificar como están distribuidas estas actividades en el tiempo y en el espacio.

Conocer que significado tienen estas actividades para si mismas y para su entorno.

\subsection{Tipo de evaluación.}

Grupo de estudio firma consentimiento informado para luego responder una entrevista semi- estructurada con 7 preguntas abiertas en relación con la si realización de actividades para sí mismas y 7 preguntas abiertas en relación la ausencia de realización de este tipo actividades.

Primero se realiza una breve reseña de histórica de la vida de la persona a la cual se entrevista, después de eso se procede a aplicar la entrevista, la entrevista no es unidireccional, se puede ir preguntando no solo de variables que a punten a sí o no, sino cruzar preguntas de acuerdo a al dialogo que se establezca con la entrevistada.

2 preguntas abiertas son transversal a la realización de actividades para si mismas como a la no realización de este tipo actividades.

En las preguntas se explora entorno a la forma, función y significado.

\subsection{Resultados}

1. Existen factores comunes en la historia ocupacional y en el significado de algunas temáticas en las mujeres entrevistadas que se relacionan con:

Las mujeres entrevistadas consideran importante realizar actividades para sí mismas, pero existe una incoherencia entre la importancia que le asignan y la utilización real de tiempo para este tipo de actividades.

El escaso tiempo libre, la mayoría de la mujeres, lo destina o comparte con la familia, lo que significa, hijos y padres

$75 \%$ de las mujeres entrevistadas, reconoce que no realiza actividades para si mismas, en la actualidad. Refieren tener largas jornadas que incluye trabajo dentro y fuera de la casa, la mayoría comienza el día 6:30 y lo terminan cerca de la media noche.

Solo el 25\% reconoce desarrollar actividad en su tiempo libre, yendo a la biblioteca de su comuna de manera irregular, hace 3 meses que no acude. 
$100 \%$ de las entrevistadas dan cuenta de haber realizado en algún otro momento de sus vidas, actividades para si mismas, refiriendo que la suspensión de ellas, se debe o se relaciona al aumento de la carga laboral y de las responsabilidades familiares.

$>75 \%$ señala como otro factor de abandono de este tipo actividades elegidas y percibidas como algo gratificante y liberador para ellas, que finalmente se transforman en una obligación más.

$>$ Si bien, la mayoría de las mujeres entrevistadas no tienen una familia que se oponga a este tipo de actividades, tampoco existe una actitud solidaria y facilitadora para que las mujeres realicen este tipo de actividades.

Dentro de las actividades que las mujeres entrevistadas elegirían y clasificarían para sí mismas están las actividades deportivas y del área intelectual.

$>$ Las mujeres concluyen que este tipo de actividades serian de gran significancia en sus vidas, porque consideran que inciden beneficiosamente en su salud y en su calidad de vida, además de aportar a su desarrollo y satisfacción personal.

2. Existen Factores diferenciadores en la historia ocupacional y el significado de algunas temáticas en las mujeres entrevistadas que se relacionan con:

Uno de los factores diferenciadores entre las entrevistadas es que un grupo de ellas se siente realizada por su trabajo. asignado a esta actividad la categoría de actividades para si mismas. Versus otro grupo que considera el trabajo como una obligación o una necesidad dentro de sus ocupaciones diarias. Esto tiene mucha relación con que una de las entrevistadas trabaja porque le gusta y se siente muy realizada con su trabajo y otra entrevistada refiere que es la única manera de equilibrar o mantener económicamente el hogar.

$>$ Otro factor diferenciador entre las entrevistadas es que un grupo de ellas también define el estar con la familia (hijos, esposo etc.) como una actividad para sí misma. Versus otro grupo que puede definir claramente actividades que apunten a satisfacer necesidades y habilidades personales. 


\section{CONCLUSIONES.}

No tan solo de las encuestas de donde surgió nuestro tema de estudio, sino más bien de la fuente de nuestras encuestadas, se pudo constatar un fuerte desequilibrio ocupacional de estas cuatro mujeres consultadas.

Estas mujeres deben hacerse cargo de múltiples responsabilidades, existe un desequilibrio de su patrón ocupacional, el trabajo, la familia, los hijos, no dan cabida o no son congruentes a las actividades para si mismas, Si bien la mayoría de las mujeres le asignan el significado de actividades, beneficiosas, necesarias y las reconoce como importantes, les cuesta darle un significado que vaya en relación directa con lo que realmente experimentan al realizarla, ya que no están realizando y están postergado este tipo de actividades en sus vidas, en donde la familia y el trabajo es lo prioritario.

Esta situación se convierte en un factor de riesgo ocupacional para estas mujeres, teniendo como consecuencia a la alineación ocupacional que se entiende como "La perdida de oportunidades o recursos para permitir el sentido o el enriquecimiento, Ejemplo de estos seria la eliminación de las ocupaciones culturales, restricciones de la creatividad de cada uno y la participación en ocupaciones monótonas y mundanas"(12) esto pudiéndose expresar en la persona a través de diferentes niveles de ansiedad, aburrimiento y agotamiento, depresión, fatiga, trastornos del sueño etc. Característica que frecuentemente se identifica con estrés.

Por lo que el estrés esta relacionado a la enfermedad como resultado de la enajenación, depravación y desequilibrio ocupacional, seria importante considerar para estudios posteriores la conciencia y percepción individual o colectiva sobre la relación que tienen estas mujeres y/o su familia acerca de sus ocupaciones y su mala o buena salud, o ampliar el estudio a conocer los niveles de satisfacción ocupacional de las mujeres en relación a las ocupaciones que desempeñan.

Si bien la desigualdad de oportunidades que ha caracterizado las ocupaciones de las mujeres ha disminuido en sociedades post industrializadas, estas desigualdades aun sigue presentes en nuestra sociedad lo cual puede ser resultado de la economía e ideas culturalmente dominantes, tales como la justicia social e igualdad, individualismo y familia, observándose aun un prejuicio de genero ocupacional, donde la mujer gana estatus como esposa y madre desde su hogar y aun pasando a terreno de lo laboral es en la familia como madre, donde encuentra mayor reconocimiento. Entonces para nuestro 
estudio aun sigue siendo una interrogante ¿Donde las mujeres como individuos diferentes expresan o satisfacen sus propias necesidades de bienestar individual?

El aporte que puede entregar este estudio piloto de investigación, es plantear estudio mas amplios de recolección de las necesidades de nuestras mujeres, en relación con las actividades para si mismas, plantear a nivel primario de salud, programas de educación sobre que son las actividades para mismas, y enseñar a las mujeres chilenas a delegar y darle importancia a las actividades que las autogratifiquen y le beneficien en su calidad de vida, además seria importante incluir en estos programas a la familia.

\section{Referencias Bibliográficas.}

1.ADIMARK. Estudio de, Encargado por Clínica las Condes y Comunidad mujer, Santiago de Chile, Julio 2007.

2.Carlos Catalán. "El uso del tiempo y el consumo de medios" (Proyecto Fondecyt 1980857)

3.Yerxa y cols. An introduction to occupational science: A foundation for occupational therapy in the 21 st century. Occupational Therapy in Health Care, 1-17 1990;

4. Townsend, E. Wilcock. A. Occupational justice. In Christiansen, C. Introduction to occupation (pp. 243-273). Prentice-Hall 2002.

5.Salazar V. Gabriel. La mujer del “ bajo pueblo” en Chile: bosquejo histórico. Revista Proposiciones $n^{\circ} 21$. Sur, Santiago, 1992

6.Salinas A., C. Las chilenas de la Colonia. Virtud sumisa, amor rebelde. LOM, Santiago 1994.

7.Entrevista a Sonia Montecinos, sección, Artes y Letras Diario, El Mercurio, Agosto 2007.

8.Larson, Word y F. Clark. Ciencia Ocupacional: desarrollo de la ciencia y la práctica de la ocupación a través de una disciplina académica”. AJOT. 45, 300-310. 1991

9.David L. Nelson . This Article was accepted for publication FAOTA December 28, 1987.

10. Larson, Word y F Clark. Desarrollo de una disciplina académica: ciencia de la ocupación. American Journal of Occupational Therapy, 45, 300-310. 1991

11. ADIMARK. Estudio realizado por la Clínica Las Condes y Comunidad Mujer "Distribución de Roles y Stress" Santiago, Julio 2007.

12. Townseend, E. Wilcock, A. Justicia Occupational. En Christiansen, C. Introducción a la ocupation (pp. 243-273). Prentice-Hall 2002. 


\section{Bibliografía Complementaria:}

Elias, N. y E. Dunning. Deporte y ocio en el proceso de la civilización. México, DF: Fondo de Cultura Económica. 1995

Elias, Norbert. Sobre el tiempo. Madrid: Fondo de Cultura Económica. 1989.

ENCLA99 (Encuesta laboral 1999). 2000. Santiago: Dirección del Trabajo, Departamento de Estudio.

ILO (International Labour Organization). "Working, longer, Working better?” The Magazine of ILO: Index. World of work $\mathrm{n}^{\mathrm{o}} 31$ (setp-october) 1999.

Izquierdo, Jordi. "El tiempo libre y las nuevas ocupaciones sociales". La Factoría n ${ }^{\circ}$ 3, disponible en: http://www.lafactoriaweb.com/articulos 1997.

Janne, Henri. "Moral de trabajo y moral de ocio: un nuevo tipo humano en perspectiva". En $\mathrm{La}$ civilización del ocio. Madrid: Guadarrama. 1968.

Larraín, J.. Modernidad razón e identidad en América Latina. Santiago: Ed. Andrés Bello. 1996

Sharim, D. y U. Silva.. "Familia y reparto de responsabilidades". Documento $n^{0} 58$. Santiago: Servicio Nacional de la Mujer (Sernam). 1998 